\title{
Chamfer or shoulder preparation can be used for metal- free polymer crowns on anterior and posterior teeth
}

\author{
Do the location of and design used when preparing metal-free polymer crowns \\ influence their survival and complication rate?
}

\section{Rammelsberg P, Spiegl K, Eickemeyer G, Schmitter M. Clinical performance of metal-free polymer crowns after 3 years in serv- ice. J Dent 2005; 33:517-523}

Design This was a randomised controlled trial conducted in Germany. Intervention Subjects were randomly allocated to two crown-preparation designs, a chamfer or shoulder finishing line. The minimum occlusal and axial reduction was $1 \mathrm{~mm}$ and $0.5 \mathrm{~mm}$, respectively. Build-ups were made with a composite material, using the corresponding dentine adhesive. After making impressions with polyether material, polymer crowns were manufactured on stone dies and adhesively luted with resin cement.

Outcome measure Follow-up appointments were scheduled after 1 month, 1 year, 2 years and 3 years. Outcome measures included failures and other complications. The aesthetic and functional performance was evaluated subjectively by visual rating scales (VAS), from 0 (completely inadequate) to 10 (perfect).

Results Seventy-one patients had a total of 114 (68 posterior and 46 anterior) single crowns prepared. The dropout rate was less than $5 \%$ per year, giving a sample of 100 crowns for the 3-year follow-up. During the 3-year observation period, 10 complications occurred, including three total fractures, three partial fractures and three decementations. Only four crowns (three total fractures and one partial fracture) had to be replaced, whereas two partial fractures could be repaired and all loosened crowns could be recemented. A Kaplan-Meier survival curve based on total failures indicated a probability of survival of $96 \%$ after 3 years. It revealed no significant effect of the location or the design used in crown preparation on the complication rate, however. Aesthetic and functional evaluation by the patients revealed a high acceptance of single polymer crowns, indicated by medians of 9-10 on the VAS.

Conclusions From this 3-year observation period, it can be concluded that $0.5 \mathrm{~mm}$ chamfer and shoulder preparation ensure that the stability of metal-free polymer crowns for anterior and posterior teeth is acceptable. Long-term stability and wear remain to be evaluated, however.

\section{Commentary}

The longevity of indirect restorations is an important issue but there is a shortage of data that can be applied clinically by the dental practitioner, particularly about the relative efficacy of different techniques. This prospective study sought to determine the effect of the finishing line and crown location, ie, anterior or posterior, on the performance of metal-free polymer crowns (Artglass, Heraeus Kulzer, Wehrheim, Germany). These have the benefit of requiring less axial tooth reduction than all ceramic or metal-ceramic crowns.

A total of 71 patients were recruited, receiving a total of 114 crowns. Details of the inclusion criteria and other clinical findings were sparse. The finishing line for the crowns was either a chamfer or shoulder of $0.5 \mathrm{~mm}$, with the preparations made by six dentists who had been trained in standardised techniques. No measurements were made of the preparations or the crowns.
Ten crowns were lost to follow-up over 3 years indicating, once again, the difficulties in prospective trials where extended periods of observation are essential. The major complication was complete or partial fracture of the crowns, which is characteristic of brittle materials such as ceramic, or highly cured polymeric materials. The survival of $96 \%$ at 3 years is of the same order as survival of two types of modern all-ceramic crown. ${ }^{1,2}$ There was no statistical evidence that the type of finishing line or crown location influenced the results, although the Kaplan-Meier curves showed a slightly higher complication rate for the chamfer compared with the shoulder group. The latter finding is supported by a laboratory-based study of polymer/ fibre-reinforced composite crowns. ${ }^{3}$

Appearance and function of the crowns were assessed as highly satisfactory by the patients using a VAS Scale at each recall visit. No information was provided about the question(s) in the VAS.

The conclusions are accurate: stability with either finish line was considered "acceptable". There were no data, however, relating to the preparations actually produced and nothing to indicate that Artglass crowns are a useful substitute for other types of indirect aesthetic restoration in anything other than the short-term. Three years is not long in fixed prosthodontics ${ }^{4}$ and this study indicates only that polymer crowns have potential.

\section{Practice point}

- This study shows the potential of polymer crowns in the short term but longer term performance needs to be evaluated.

\section{Richard lbbetson}

Postgraduate Dental Institute, Edinburgh, Scotland, UK

1. McLaren EA, White SN. Survival of In-Ceram crowns in a private practice: a prospective clinical trial. J Prosthet Dent 2000; 83:216-222.

2. Fradeani $M, D^{\prime}$ Amelio $M$, Redemagni $M$, Corrado M. Five year follow-up with Procera all-ceramic crowns. Quintessence Int_2005; 36:105-113.

3. Cho L, Choi J, Yi YJ, Park CJ. Effect of finish line variants on marginal accuracy and fracture strength of ceramic optimized polymer/ fiber-reinforced composite crowns. J Prosthet Dent 2004; 91:554-560.

4. Donovan T, Simonsen RJ, Guertin G, Tucker RV. Retrospective clinical evaluations of 1314 cast gold restorations in service for 1 to 52 years. J Esthet Restor Dent 2004; 16:194-204.

Evidence-Based Dentistry (2006) 7, 30

doi:10.1038/sj.ebd.6400403 